11. Setala A, Gittelsohn J, Speakman K et al. (2011) Linking farmers to community stores to increase consumption of local produce: a case study of the Navajo Nation. Public Health Nutr 14, 1658-1662.

12. Chiang P-H, Wahlqvist ML, Lee M-S et al. (2011) Fast-food outlets and walkability in school neighbourhoods predict fatness in boys and height in girls: a Taiwanese population study. Public Health Nutr 14, 1601-1609.

13. Jilcott SB, Wade S, McGuirt JT et al. (2011) The association between the food environment and weight status among eastern North Carolina youth. Public Health Nutr 14, 1610-1617.

14. Svastisalee CM, Nordahl H, Glümer C et al. (2011) Supermarket and fast-food outlet exposure in Copenhagen: associations with socio-economic and demographic characteristics. Public Health Nutr 14, 1618-1626.

15. Campbell AA, Akhter N, Sun K et al. (2011) Relationship of homestead food production with night blindness among children below 5 years of age in Bangladesh. Public Health Nutr 14, 1627-1631.

16. Rydén PJ \& Hagfors L (2011) Diet cost, diet quality and socio-economic position: how are they related and what contributes to differences in diet costs? Public Health Nutr 14, 1680-1692.

17. Katz DL, Doughty K, Niike V et al. (2011) A cost comparison of more and less nutritious food choices in US supermarkets. Public Health Nutr 14, 1693-1699.

18. D'Angelo H, Suratkar S, Song H-J et al. (2011) Access to food source and food source use are associated with healthy and unhealthy food-purchasing behaviours among low-income African-American adults in Baltimore City. Public Health Nutr 14, 1632-1639.

19. Thompson JL, Bentley G, Davis M et al. (2011) Food shopping habits, physical activity and health-related indicators among adults aged $\geq 70$ years. Public Health Nutr 14, 1640-1649.

\title{
Vitamin D in the prevention of disease - what evidence do we still need?
}

During recent decades interest in the health effects of vitamin D has increased enormously, as described in the review included in this issue of Public Health Nutrition ${ }^{(1)}$. The demand for a change in dietary recommendations for vitamin $\mathrm{D}$ has received much attention, and resulted in new recommendations in the USA ${ }^{(2)}$.

The new era of vitamin D research started in the 1960s when the metabolism and metabolites of vitamin D were revealed, including the most important metabolite, 25-hydroxyvitamin D (25OHD), produced in the liver and the active one, 1,25-dihydroxyvitamin $\mathrm{D}\left(1,25-(\mathrm{OH})_{2} \mathrm{D}\right.$, calcitriol), synthesised in the kidneys. Both are found in the circulation. Furthermore, the vitamin D receptor (VDR) was found and its role as a transcriptional factor, when activated by calcitriol, was discovered and described. During the years to follow, researchers showed that the vitamin D receptor was present in almost all cells of the human body and that many of the cell types studied also had the machinery, i.e. 1-hydroxylase, to produce calcitriol. Recently it has been shown that e.g. osteoblasts are able to produce calcitriol locally. Over the years it has been shown that, on the cellular level, calcitriol is involved in the regulation of many physiological events. Thus, the active form of vitamin D is important or crucial for e.g. intestinal calcium absorption, immunomodulation and insulin production ${ }^{(3)}$.

Although there seems to be strong evidence of a role for calcitriol in many physiological events, there are very few, if any, studies showing a connection between serum calcitriol concentrations and health outcomes on an individual or population level, with the exception of renal disease. However, serum 25-OHD concentrations are associated with a number of health outcomes. This is fascinating and could, at least in some instances, be explained by the fact that 25-OHD serves as a substrate for locally produced calcitriol - if the 25-OHD concentration is low and there is not enough substrate for the production of calcitriol.

The Institute of Medicine (IoM) published its revised dietary recommendations for vitamin $\mathrm{D}$ and calcium at the end of $2010^{(2)}$. This is the first time that IoM recommendations have been based on systematic review of scientific evidence alone, and in this case they were largely based on two commissioned systematic reviews ${ }^{(4,5)}$. The new recommendations have been criticised for many reasons, e.g. not making the procedure transparent, not allowing the referees to comment on the final recommendations, and not taking all health outcomes into account. This criticism was also brought forward in the eight invited Letters to the Editor in the April issue of this journal, such as that by Boucher ${ }^{(6)}$. The first of the commissioned reviews focused on bone health, and the second on many other health outcomes including bone health; both were based mainly on randomised controlled trials (RCT) but included also observational, cohort and case-control studies. RCT are considered to be the gold standard for proving an 
effect of a substance (in this case vitamin D) on a specific outcome. The problem is that all RCT regarding vitamin D have focused on bone health and fractures, mostly in the elderly. Moreover, these RCT usually were not designed to find an optimal dose, but to show effects of one dose, either $10 \mu \mathrm{g}$ or $20 \mu \mathrm{g}$ vitamin $\mathrm{D} / \mathrm{d}$. This makes it difficult to draw conclusions regarding optimal intakes from these RCT. Moreover, a calcium supplement has often been included in the $\mathrm{RCT}^{(4)}$. Indicative of the available evidence is the conclusion in the review by Chung et al. $^{(5)}$ :

... the majority of the findings concerning vitamin

$\mathrm{D}$, calcium and or combination of these on the health outcomes were inconsistent. Synthesizing a dose-response relation between intake of either vitamin $\mathrm{D}$, calcium or both and health outcomes proves challenging.

An important report by the International Agency for Research on Cancer (IARC) focused on vitamin D and cancer $^{(7)}$. The Agency did not find enough evidence for any relationship between cancers other than colorectal cancer and vitamin D status (and this with reservations). The IARC report ended up with seven, very wise, overall conclusions, including the following:

The epidemiological evidence from observational studies for an inverse association between serum 25-hydroxyvitamin D levels and the incidence of colorectal cancer and sporadic colorectal adenoma was consistent and persuasive. There is, however, only limited evidence of a causal link due to possible confounding by other dietary or lifestyle factors.

No data exists on the health effects of intakes of high doses of vitamin $\mathrm{D}$ (say, $30 \mu \mathrm{g}$ per day or more) over the long term.

There is no data available on the health hazards of long-term maintenance of high 25-hydroxyvitamin $\mathrm{D}$ serum levels in healthy subjects over long periods.

Hypotheses on vitamin D status and colorectal cancer, cardiovascular diseases and all-cause mortality should be tested in appropriately designed randomised controlled trials.

Although the IARC review is from 2008 the message is still relevant. Recently, Mason and co-workers ${ }^{(8)}$ concluded that 'the existing evidence falls short' [as regards vitamin D and prevention of cancer] and calls for specific RCT.

In this issue, Scragg $^{(1)}$ has directed his interest on the relationship of vitamin $\mathrm{D}$ and common diseases and mortality in persons older than 50 years in cohort studies. He has summarised the relationship between vitamin D and main health outcomes, concluding that baseline 25-OHD concentrations predict increased risk of fractures, colorectal cancer, CVD and all-cause mortality. The associations are weak in these cohort studies and, as the author points out, could be caused by confounders such as obesity and physical activity. Obese persons have, on average, lower 25-OHD concentrations, and low 25-OHD concentrations could indicate a low physical activity, which is a risk factor for many diseases. The last part of the review focuses on a very important issue, vitamin D status and mortality, especially all-cause mortality, which was neglected in the IoM report although it was presented in the systematic review by Chung et $a l^{(5)}$. Recently, some reports have shown that both low and high serum 25-OHD concentrations are associated with increased mortality ${ }^{(9,10)}$.

To conclude, almost all reviews, reports and comments related to vitamin $\mathrm{D}$ and health outcomes make a plea for RCT with higher doses of vitamin D for longer periods of time. As vitamin D status is influenced by a number of confounding factors this is the only way to get a more definitive answer to the question of whether or not vitamin D is related to all or some of the abovementioned health outcomes, as well as to the question of the optimal dose. A longer time frame for RCT is, however, challenging, as compliance is of utmost importance in long-term trials. Scragg ${ }^{(1)}$ has focused on adults in his request for RCT, but there is also a need for studies in children and adolescents. The research task aiming at getting answers to these questions, which could form the basis for new dietary recommendations, is huge. There are already some larger RCT ongoing or starting, but more studies are needed. It is hoped that funding bodies will give highest priority to this important line of research.

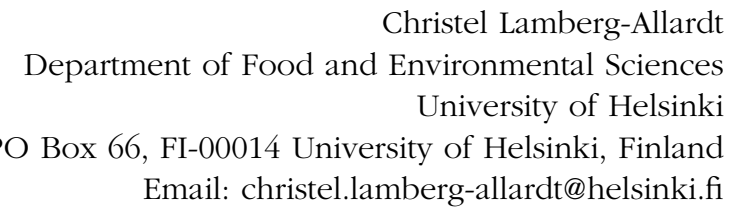

\section{References}

1. Scragg R (2011) Vitamin D and public health: an overview of recent research on common diseases and mortality in adulthood. Public Health Nutr 14, 1515-1532.

2. Institute of Medicine (2011) Dietary Reference Intakes for Calcium and Vitamin D. Washington, DC: National Academies Press.

3. Holick MF (2007) Vitamin D deficiency. N Engl J Med 357, 266-281.

4. Cranney A, Horsley T, O'Donnell S et al. (2007) Effectiveness and Safety of Vitamin D in Relation to Bone Health. Evidence Report/Technology Assessment no. 158 (Prepared by the University of Ottawa Evidence-based Practice Center (UO-EPC) under Contract No. 290-02-0021). AHRQ Publication no. 07-E013. Rockville, MD: Agency for Healthcare Research and Quality.

5. Chung M, Balk EM, Brendel M et al. (2009) Vitamin D and Calcium: Systematic Review of Health Outcomes. Evidence Report/Technology Assessment no. 183 (Prepared by Tufts Evidence-based Practice Center under Contract 
No. 290-2007-10055-I). AHRQ Publication no. 09-E015. Rockville, MD: Agency for Healthcare Research and Quality.

6. Boucher BJ (2011) The 2010 recommendations of the American Institute of Medicine for daily intakes of vitamin D. Public Health Nutr 14, 740.

7. International Agency for Research on Cancer (2008) Vitamin D and Cancer. IARC Working Group Reports no 5. Lyon: IARC; available at http://www.iarc.fr/en/ publications/pdfs-online/wrk/wrk5/Report_vitD.pdf
8. Manson JE, Mayne ST \& Clinton SK (2011) Vitamin D and prevention of cancer - ready for prime time? $N$ Engl J Med 364, 1385-1387.

9. Autier P \& Gardini S (2007) Vitamin D supplementation and total mortality: a meta-analysis of randomized controlled trials. Arch Intern Med 167, 1730-1737.

10. Michaëlsson K, Baron JA, Snellman G et al. (2010) Plasma vitamin $\mathrm{D}$ and mortality in older men: a community-based prospective cohort study. Am J Clin Nutr 92, 841-848. 